\title{
The effect of laryngoscopy and intubation on the qNOX and qCON in spinal surgery
}

Gritsan A., Dovbysh N., Korchagin E., Chernetsky O. Krasnoyarsk Regional Clinical Hospital, Krasnoyarsk

\section{State Medical University, Krasnoyarsk, Russian Federation}

During the past 10 years there have been several analgesia monitors, which can be divided according to the principle of action into 2 groups

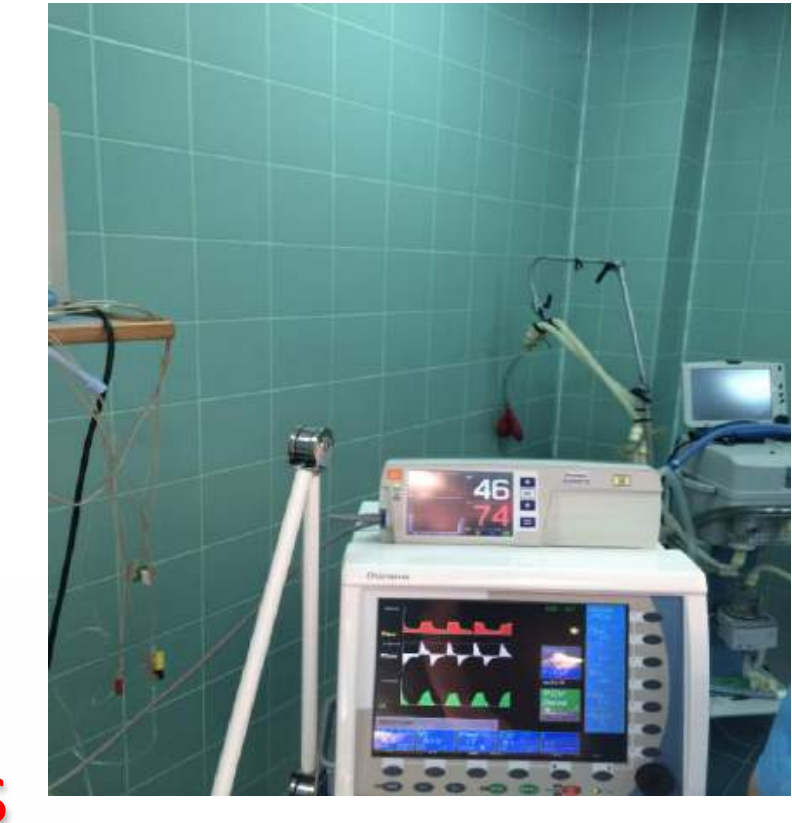

Registration and analysis of brain signals

- electroencephalography (EEG) and auditory evoked potentials (AEP)
The acquisition parameters of the autonomic nervous.

Register of qNOX based on the principle of adaptive network based fuzzy inference system.

Goal of study: To evaluate the information value of the qNOX and qCON on stressful effect of laryngoscopy and intubation

Materials and methods: data was recorded from 12 patient -8 men and 4 women, age 40.6 9 9.6 (I-II class according ASA), who were spinal syrgery. Surgical intervention took place under general anesthesia. Induction was carried out on the basis of propofol $2.8 \pm 0.7 \mathrm{mg} / \mathrm{kg}$ and fentanyl $2.5 \pm 0.4 \mu \mathrm{g} / \mathrm{kg}$, maintenance - on the basis of sevoflurane 0.7 to $1.1 \mathrm{MAC}$ and fentanyl $1.8 \pm 0.3 \mu \mathrm{g} / \mathrm{kg}$. Before and after laryngoscopy and intubation were evaluated hemodynamic parameters (monitor Phillips MP 20): SP, DP, MAP, HR, assessment of the qNOX and qCON (monitor ICARDQ Chirana); the presence of go and the lack of motor response to laryngoscopy and intubation. The level of qCON and qNOX was considered adequately in the range of 40-60. Statistical analysis was performed using the $\mathrm{t}$-test for dependent samples, considering a statistically significant change in $\mathrm{p}$ value $<0.05$.

Results and discussion: the values of the qNOX and qCON decreased to the target values $43.6 \pm 15.4$ and $44.4 \pm 9.5$ respectively after administration of propofol and fentanyl before conducting laryngoscopy and intubation (see table).

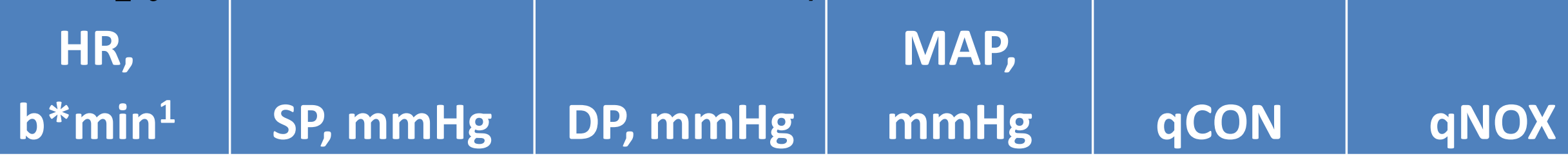

before laryngoscopy and

intubation

\begin{tabular}{|l|l|l|l|l|l|}
$89.8 \pm 15.6$ & $123 \pm 23,4$ & $76.1 \pm 15.6$ & $91.7 \pm 16.3$ & $44.4 \pm 9.5$ & $43.6 \pm 15.4$
\end{tabular}

after laryngoscopy and

intubation

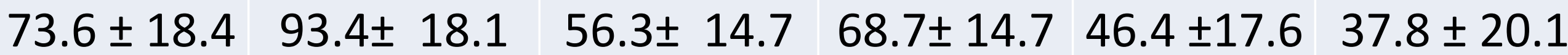

\begin{tabular}{l|l|l|l|l|l|}
$p<0.05$ & $p<0.05$ & $p<0.05$ & $p<0.05$ & $p=0.2$ & $p=0.15$
\end{tabular}

Laryngoscopy and intubation did not cause pressor hemodynamics reactions and even there were statistically significant decrease of the estimated hemodynamic parameters, which can be attributed to the relative hypovolemia and the patients are on his side, when non-invasive BP was measured on the raised hand.

Statistically significant changes qNOX and qCON after laryngoscopy and intubation did not happen.

Conclusion: Focus on the values of the qNOX and qCON allows to prevent the decreased of the flow of inhaled anesthetics in the future maintenance 